\title{
Efecto de la lluvia en el comportamiento de los bordos del Río de la Compañía
}

\author{
J. Alberro-Aramburu, R. Hernández-Hernández \\ Instituto de Ingeniería, UNAM \\ E-mail:rhh@pumas.iingen.unam.mx
}

(recibido: septiembre de 2002; aceptado: marzo de 2003)

\begin{abstract}
Resumen
Durante la estación de lluvias del año 2001, se notaron movimientos horizontales apreciables en el terraplén de la margen izquierda del Río de la Compañía, no así en el terraplén de su margen derecha. El presente artículo pretende explicar esta diferencia de comportamiento mediante la consideración del efecto diferenciado de la lluvia en los bordos izquierdo y derecho del canal.
\end{abstract}

Descriptores: flujo transitorio, lluvia, terraplén, deformaciones.

\section{Abstract}

During the wet season of 2001, horizontal movements are observed in the left border, but no so in the right border of the rio de la Compañia. This paper, try to explain this difference of behaviour, taking into account the differential effect of the rain on each border.

Keywords: transient flow, rain, earthfill, strains.

\section{Introducción}

Durante la estación de lluvias del año 2001, se notaron movimientos apreciables en los terraplenes del canal del Río de la Compañía. En particular, en la zona aledaña a la que había sufrido una falla en su bordo izquierdo durante la estación de lluvias del año 2000 (cad 4 +296 al $4+456$ ), el desplazamiento máximo horizontal medido fue de $36 \mathrm{~mm}$ hacia el SW (CFE, 2001). Tal desplazamiento se presentó en la corona del terraplén que constituye el bordo izquierdo del canal. En el bordo derecho y a lo largo de los mencionados cadenamientos los desplazamientos hacia el SW fueron prácticamente nulos.

El presente informe pretende explicar esta diferencia entre los movimientos horizontales medidos en los terraplenes que conforman los bordos izquierdo y derecho del canal del Río de la Compañía. Podría pensarse que tal diferencia es causada por la heterogeneidad en la estratigrafía del subsuelo bajo los terraplenes, sin embargo, los sondeos efectuados en el sitio no muestran claramente tales heterogeneidades. Asimismo, el ascenso del nivel de agua en el canal ocasiona un incremento de los desplazamientos en los bordos. No obstante, tal incremento debería ser, contrariamente a lo observado, idéntico en los bordos izquierdo y derecho con geometría y materiales constitutivos idénticos.

Hemos de considerar entonces, el efecto diferenciado de la lluvia en los bordos izquierdo y derecho del canal, a fin de tratar de explicar las observaciones. 


\section{Intensidad e inclinación respecto a la vertical de la lluvia}

Se considera una lluvia de intensidad e en metros/día. La inclinación respecto a la vertical de la lluvia impulsada por el viento se supone igual a $\beta$ (figuras 1.a y 1.b)

En esas condiciones el volumen de agua que penetra en el talud externo del bordo izquierdo (Figura 1.a) por unidad de tiempo y por unidad de longitud es,

$$
d v_{i}=e\left(d l^{\prime}\right)=e(d l) \cos (\beta-\gamma)=e \frac{\cos (\beta-\gamma)}{\cos (\gamma)} d x
$$

Idénticamente para el bordo derecho,

$$
d v_{d}=e\left(d l^{\prime}\right)=e(d l) \cos (\beta+\gamma)=e \frac{\cos (\beta+\gamma)}{\cos (\gamma)} d x
$$

En consecuencia, dependiendo de la inclinación de la lluvia respecto al talud externo del bordo, ésta tendrá efectos diferentes sobre el comportamiento del mismo. En particular,

si $\quad(\beta+\gamma)=\frac{\pi}{2}\left\{\begin{array}{c}d v_{i}=2(e) \operatorname{sen}(\gamma) \\ d v_{d}=0\end{array}\right.$

Para un valor de $\gamma$ igual con $26.5^{\circ}(\tan \gamma=0.5)$, que se considera representativo para los bordos del canal de la Compañía, se tiene cuando

$$
\beta=\frac{\pi}{2}-\gamma=62.5^{\circ}\left\{\begin{array}{c}
d v_{i}=0.9(e) \\
d v_{d}=0
\end{array}\right.
$$

En estas condiciones, sólo el bordo izquierdo registrará los efectos de la lluvia.

\section{Ecuación diferencial básica.}

Determinación de la línea superior de corriente con lluvia

Se considera válida la hipótesis de Dupuit de la casi-verticalidad de las equipotenciales durante el flujo transitorio. El terreno se considera homogéneo, isótropo e indeformable. Se analiza el caso del bordo izquierdo del canal de la Compañía sometido a una lluvia con inclinación $\beta$.

En esas condiciones, la ecuación diferencial que rige el equilibrio de los gastos de entrada y salida de agua de un prisma de paredes verticales (Figura 2) limitado en su frontera superior por la superficie libre de escurrimiento y en su frontera inferior por un estrato impermeable es:

$$
d q=d\left[-k \frac{d h}{d x} h\right] d t=e \frac{\cos (\beta+\gamma)}{\cos (\gamma)} d x d t-n d h d x
$$

o sea

$$
\frac{d q}{d x}=\frac{d}{d x}\left[-k \frac{d h}{d x} h\right] d t=e \frac{\cos (\beta+\gamma)}{\cos (\gamma)} d t-n d h
$$

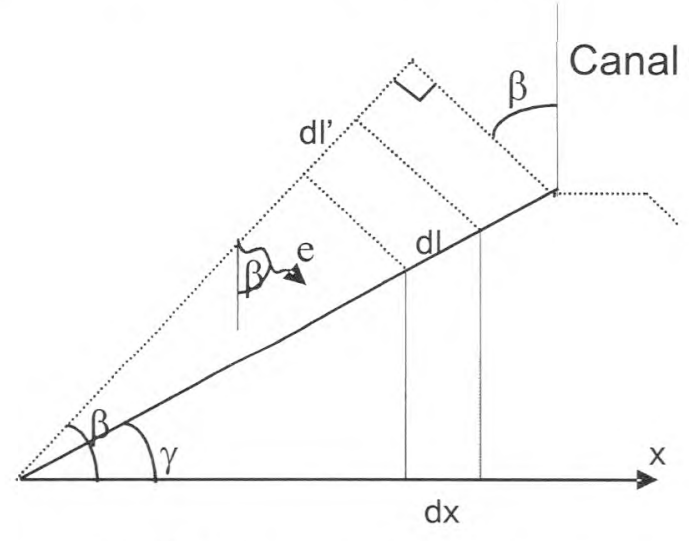

Talud externo del bordo izquierdo.

a)

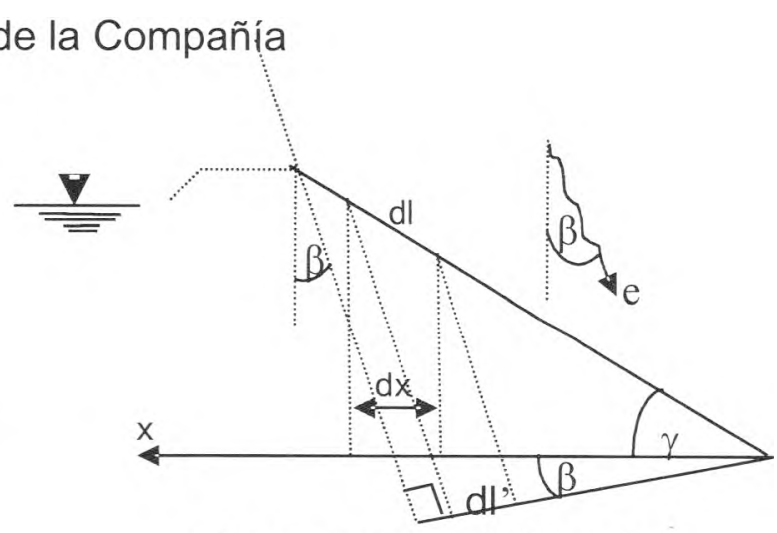

Talud externo del bordo derecho.

b)

Figura 1 
Con $k$, permeabilidad del medio [m/día].

$e$, intensidad de la lluvia [m/día].

$\mathrm{n}$, porosidad del material constitutivo del bordo.

La ecuación (1) se escribe,

$$
k \frac{\partial^{2} h^{2}}{\partial x^{2}}+2 e \frac{\cos (\beta-\gamma)}{\cos (\gamma)}=2 n \frac{\partial h}{\partial t}
$$

Esta ecuación es de segundo grado en x y no lineal. Se lineariza de acuerdo con lo propuesto por Polubarinova (1962), tomando como incógnita la función,

$$
G=h^{2}
$$

La ecuación (2) se transforma, por tanto, en:

$$
k \frac{\partial^{2} G}{\partial x^{2}}+2 e \frac{\cos (\beta-\gamma)}{\cos (\gamma)}=\frac{n}{\sqrt{G}} \frac{\partial h}{\partial t}
$$

y se aproxima el valor de $\sqrt{G}$ mediante la igualdad:

$$
\sqrt{G} \approx \frac{h_{1}+h_{2}}{2}=h_{t m}
$$

En consecuencia, la ecuación diferencial básica del flujo transitorio de un talud de pendiente $\tan (\gamma)$, sometido a una lluvia de intensidad e y de inclinación $\beta$ es:

$$
h_{m} k \frac{\partial^{2} G}{\partial x^{2}}-n \frac{\partial h}{\partial t}=-2 e \frac{\cos (\beta-\gamma)}{\cos (\gamma)} h_{m}
$$

La intensidad de la lluvia y su inclinación es función del tiempo transcurrido desde el inicio de la temporada de lluvias, por lo que se considera:

$$
f(t)=2 e \frac{\cos (\beta-\gamma)}{\cos (\gamma)}
$$

En casos particulares $f(t)$ puede ser constante. La ecuación (3) se escribe finalmente:

$$
k h_{m} \frac{\partial^{2} G}{\partial x^{2}}-n \frac{\partial h}{\partial t}=-h_{m} f(t)
$$

\section{Solución de la ecuación diferencial básica}

Se utiliza la transformada de Laplace de la ecuación (4), resultando:

$$
k h_{m} \frac{\partial^{2} \bar{G}}{\partial x^{2}}-n(p \bar{G}-g(x, 0))=-\bar{f} h_{m}
$$

Con $\bar{G}=\bar{G}(x, p)$ transformada de Laplace de la función $\mathrm{G}(x, t)$

\section{Bordo izquierdo}

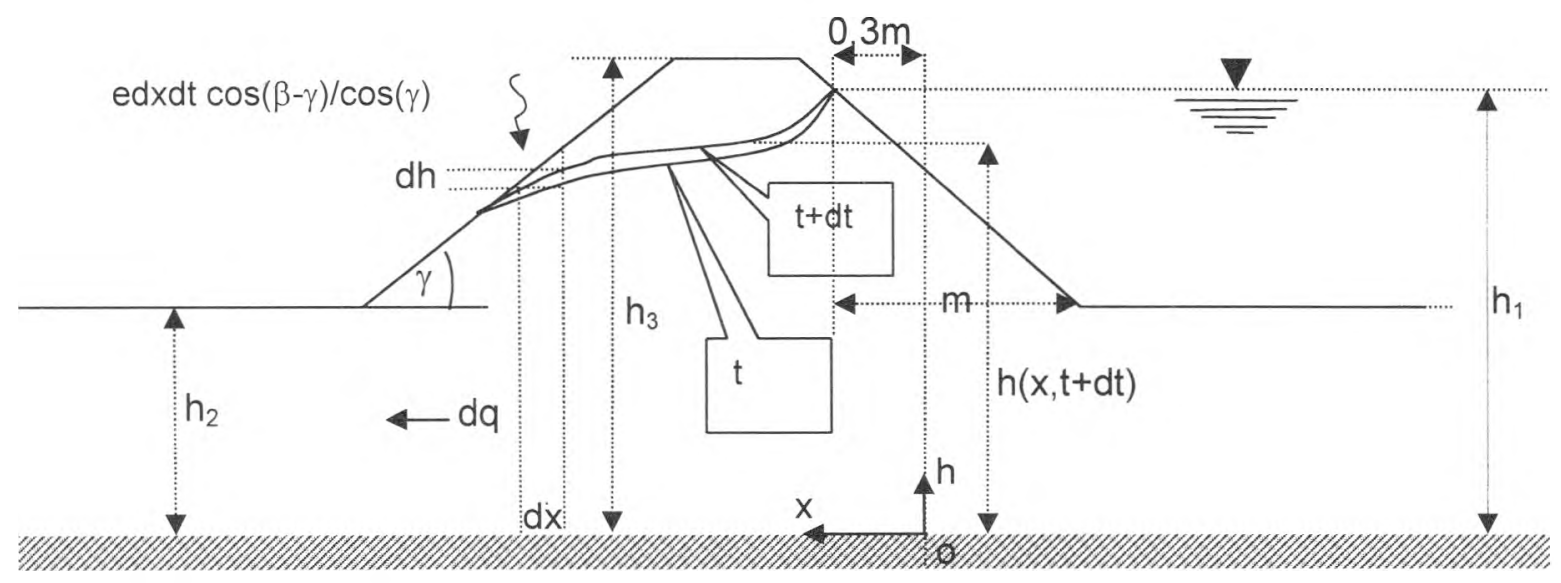




$$
G(x, 0)=\left[h_{1}^{2}-\left(h_{1}^{2}-h_{2}^{2}\right) \frac{X}{L}\right]
$$

ecuación de la parábola de Kozeny que representa la línea superior de flujo inicial.

$$
\begin{aligned}
& \bar{f}(p)=\text { Transformada de Laplace de la función } \\
& F(t) .
\end{aligned}
$$

La ecuación sin segundo miembro correspondiente es:

$$
k h_{m} \frac{\partial^{2} \bar{G}}{\partial x^{2}}-n p \bar{G}=0
$$

Cuya solución general es

$$
\bar{G}=C_{1} e^{-\sqrt{\frac{n p}{k h_{m}} x}}+C_{2} e^{\sqrt{\frac{n p}{k h_{m}} x}}
$$

Una solución particular de la ecuación con segundo miembro es:

$$
\bar{G}=A x+B
$$

con

$$
-n p(A x+B)=-n\left[h_{1}^{2}-\left(h_{1}^{2}-h_{2}^{2}\right) \frac{X}{L}\right]-h_{m} \bar{f}
$$

Por lo que

$$
A=-\frac{h_{1}^{2}-h_{2}^{2}}{L p} \quad B=\frac{h_{1}^{2}}{p}+\frac{h_{m} \bar{f}}{n p}
$$

La solución de la ecuación (5) es,

$$
\begin{gathered}
\bar{G}=C_{1} e^{-\sqrt{\frac{n p}{k h_{m}} x}}+C_{2} e^{\sqrt{\frac{n p}{k h_{m}} x}}+ \\
{\left[h_{1}^{2}-\left(h_{1}^{2}-h_{2}^{2}\right) \frac{X}{L}\right] \frac{1}{p}+\frac{h_{m} \bar{f}}{n p}}
\end{gathered}
$$

Para definir las constantes $\mathrm{C}_{1}$ y $\mathrm{C}_{2}$ se utilizan las condiciones de frontera:

$$
\bar{G}(0, p)=\int_{0}^{\infty} G(0, t) e^{-p t} d t=\frac{h_{1}^{2}}{p} \text { pues } G(0, t)=h_{1}^{2}
$$

$$
\bar{G}(L, p)=\int_{0}^{\infty} G(L, t) e^{-p t} d t=\frac{h_{2}^{2}}{p} \text { pues } G(L, t)=h_{2}^{2}
$$

Que implican que de acuerdo con (6)

$$
\bar{G}(0, p)=\frac{h_{1}^{2}}{p}=\int_{0}^{\infty} G(0, t) e^{-p t} d t=C_{1}+C_{2}+\frac{h_{1}^{2}}{p}+\frac{h_{m} \bar{f}}{n p}
$$

$$
\bar{G}(L, p)=\frac{h_{2}^{2}}{p}=C_{1} e^{-\sqrt{\frac{n p}{k h_{m}}} L}+C_{2} e^{\sqrt{\frac{n p}{k h_{m p}}} L}+\frac{h_{2}^{2}}{p}+\frac{h_{m} \bar{f}}{n p}
$$

Despejando $\mathrm{C}_{1}$ y $\mathrm{C}_{2}$ resulta

$$
C_{1}=-\frac{h_{m} \bar{f}}{n p} \frac{e^{\sqrt{\frac{n p}{k h_{m}} L}}}{1+e^{\sqrt{\frac{n p}{k h_{m}} L}}} \quad C_{2}=-\frac{h_{m} \bar{f}}{n p} \frac{1}{1+e^{\sqrt{\frac{n p}{k h_{m p}} L}}}
$$

por lo que, finalmente

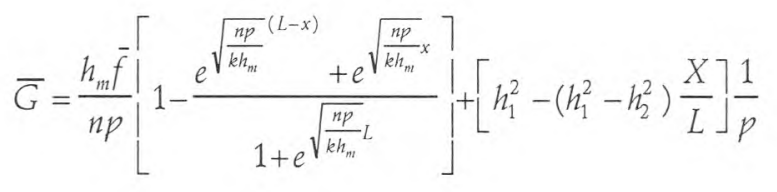

Se comprueba que

$$
\bar{G}(0, p)=\frac{h_{1}^{2}}{p} \quad \bar{G}(L, p)=\frac{h_{2}^{2}}{p}
$$

La antitransformada de $\bar{G}$ obtiene, desarrollando en serie la función

$$
\frac{1}{1+e^{\sqrt{\frac{n p}{k h_{m}} L}}}=\sum_{N=1}^{\infty}(-1)^{(N-1)} e^{-N \sqrt{\frac{n p}{k h_{m}} L}}=
$$

$$
\sum_{N=0}^{\infty}(-1) e^{N}-(N+1) \sqrt{\frac{n p}{k h_{l t}} L}
$$

por lo que 
$\left.\bar{G}=\frac{h_{n f} \bar{f}}{n p} \mid 1-\sum_{N=0}^{\infty}(-1)^{N}\left(\begin{array}{l}e^{-(N L+x) \sqrt{\frac{m p}{k h_{n}}}} \\ +e^{-((N+1) L-x)} \sqrt{\frac{m p}{k h_{m}}}\end{array}\right)\right)+\left[\left[h_{1}^{2}-\left(h_{1}^{2}-h_{2}^{2}\right) \frac{X}{L}\right] \frac{1}{p}\right.$

Utilizando las tablas de antitransformadas proporcionadas por Carslaw y Jaeger (1959), se obtiene designando por erfc la función error complementaria:

$$
\begin{aligned}
& L^{-1}\left[\frac{e^{-(N L+x)} \sqrt{\frac{n p}{k h_{m}}}}{p}\right]=\operatorname{erfc}\left(\frac{N L+x}{2 \sqrt{\frac{k h_{m}}{n} \tau}}\right) \\
& L^{-1}\left[\frac{e^{-((N+1) L-x)} \sqrt{\frac{p p}{k h_{m}}}}{p}\right]=\operatorname{erfc}\left(\frac{(N+1) L-x}{2 \sqrt{\frac{k h_{m}}{n} \tau}}\right) \\
& L^{-1}\left(h_{m} \bar{f}\right)=h_{m} f(t)
\end{aligned}
$$

Mediante el teorema de convolución, resulta

$$
\begin{gathered}
G(x, t)=\frac{h_{m}}{n} \int_{0}^{t} f(t-\tau)\left(1-\sum_{N=0}^{\infty}\left(\Re_{1}+\Re_{2}\right)\right)(d \tau)+ \\
{\left[h_{1}^{2}-\left(h_{1}^{2}-h_{2}^{2}\right) \frac{X}{L}\right]}
\end{gathered}
$$

Donde,

$$
\begin{gathered}
\Re_{1}=\operatorname{erfc}\left[\frac{N L+x}{2 \sqrt{\frac{k h_{m}}{n} \tau}}\right] \\
\mathfrak{R}_{2}=\operatorname{erfc}\left(\frac{(N+1) L-x}{2 \sqrt{\frac{k h_{m}}{n} \tau}}\right)
\end{gathered}
$$

Integrando por partes esta expresión con

$$
U=\frac{h_{m}}{n} f(t-\tau)
$$

$$
\begin{gathered}
d V=1-\sum_{N=0}^{\infty}\left(\Re_{1}+\Re_{2}\right) d \tau \\
G=[U V]_{0}^{t}-\int_{0}^{+} V d U+\left[h_{1}^{2}-\left(h_{1}^{2}-h_{2}^{2}\right) \frac{X}{L}\right]
\end{gathered}
$$

resulta

$$
\begin{gathered}
G=\frac{h_{m}}{n}\left[\tau f(t-\tau)\left(1-\sum_{N=0}^{\infty}\left(\aleph_{1}+\aleph_{2}\right)\right)\right]_{0}^{t} \\
-\frac{h_{m}}{n} \int_{0} \frac{\partial f(t-\tau)}{\partial \tau}\left[1-\sum_{N=0}^{\infty}\left(\aleph_{1}+\aleph_{2}\right)\right] d \tau+ \\
{\left[h_{1}^{2}-\left(h_{1}^{2}-h_{2}^{2}\right) \frac{X}{L}\right]}
\end{gathered}
$$

Donde,

$$
\aleph_{1}=4 i^{2} \operatorname{erfc} \frac{N L+x}{2 \sqrt{\frac{k h_{m}}{n} \tau}}
$$

$$
\aleph_{2}=4 i^{2} \operatorname{erfc} \frac{(N+1) L-x}{2 \sqrt{\frac{k h_{m}}{n} \tau}}
$$

La serie que aparece en la expresión (7) es rápidamente convergente. En el apéndice I se presenta un programa de cálculo que permite determinar el valor de $G(x, t)$ de acuerdo con la expresión (7).

Los datos necesarios para efectuar el cálculo son:

\section{Datos geométricos}

$\mathrm{H}_{1}=$ Espesor de agua desde el nivel del embalse hasta la capa impermeable (Figura 2) en $\mathrm{m}$.

$\mathrm{H}_{2}=$ Espesor de agua desde el nivel de terreno aguas abajo del bordo hasta la capa impermeable (Figura 2) en m.

$\mathrm{H}_{3}=$ Elevación de la corona respecto a la frontera impermeable (Figura 2) en $\mathrm{m}$.

GAMMA1 = Ángulo del talud aguas abajo del bordo con la horizontal en grados.

GAMMA2 = Ángulo del talud aguas arriba del bordo con la horizontal en grados. $\mathrm{L} 0=$ Ancho de la corona en $\mathrm{m}$. 
Datos de lluvia

$\mathrm{T} 0=$ Duración de la estación de lluvias en días.

T1 = Tiempo a partir del inicio de la estación de lluvias en que se requiere calcular $G$ en días.

$\mathrm{T}(\mathrm{I})=$ Tiempo I a partir del inicio de la estación de lluvias en días.

$E(I)=$ Intensidad de la lluvia para $T(I)$ en $\mathrm{m} /$ día.

BETA(I) = Ángulo de la lluvia para $T(I)$ con la vertical en grados.

Estos tres últimos datos deben aparecer en un archivo denominado DATLLUVIA e integrado de antemano.

Datos del suelo constitutivo del terraplén

$\mathrm{K}=$ Permeablilidad en $\mathrm{m} /$ día .

$\mathrm{N}=$ Porosidad .

Si la intensidad E(I) y la inclinación de la lluvia BETA(I) son constantes:

$$
f(t-\tau)=A=\text { constante }=2 e \frac{\cos (\beta-\gamma)}{\cos (\gamma)}
$$

y la expresión (7) se reduce a

$$
G(x, t)=h^{2}(x, t)=\frac{A h_{m} t}{n}\left[1-\sum_{N=0}^{\infty}\left(\aleph_{1}+\aleph_{2}\right)\right]+
$$

$$
\left[h_{1}^{2}-\left(h_{1}^{2}-h_{2}^{2}\right) \frac{X}{L}\right]
$$

Donde,

$$
\begin{gathered}
N_{1}=4 i^{2} \operatorname{erfc} \frac{N L+x}{2 \sqrt{\frac{k h_{m}}{n} \tau}} \\
N_{2}=4 i^{2} \operatorname{erfc} \frac{(N+1) L-x}{2 \sqrt{\frac{k h_{m}}{n} \tau}}
\end{gathered}
$$

\section{Caso del talud de aguas abajo del bordo izquierdo. Canal de la Compañía}

Los datos de lluvia y geométricos necesarios para efectuar el cálculo, los cuales se consideraron representativos para el caso del talud de aguas abajo del bordo izquierdo del canal de la Compañía, son:

\begin{tabular}{ccccccc}
\hline \hline $\mathrm{H}_{1}$ & $=$ & 31.7 & $\mathrm{E}(\mathrm{l})$ & $=$ & $\begin{array}{c}0.044 \\
\mathrm{~m} / \mathrm{d}\end{array}$ \\
$\mathrm{H}_{2}$ & $=$ & 20.0 & $\mathrm{~T} 0$ & $=$ & 180 días \\
$\mathrm{H}_{3}$ & $=$ & 33.0 & $\mathrm{~T} 1$ & $=$ & 180 días \\
\hline \hline GAMMA 1 & $=$ & $26.5^{\circ}$ & $\mathrm{L} 0$ & $=$ & $6.0 \mathrm{~m}$ \\
GAMMA 2 & $=$ & $21.8^{\circ}$ & $\mathrm{K}$ & $=$ & $0.009 \mathrm{~m} /$ día \\
BETA (1) & $=$ & $63.5^{\circ}$ & $\mathrm{N}$ & $=$ & 0.4 \\
& & & $\mathrm{~A}$ & $=$ & $0.008 \mathrm{~m} /$ día
\end{tabular}

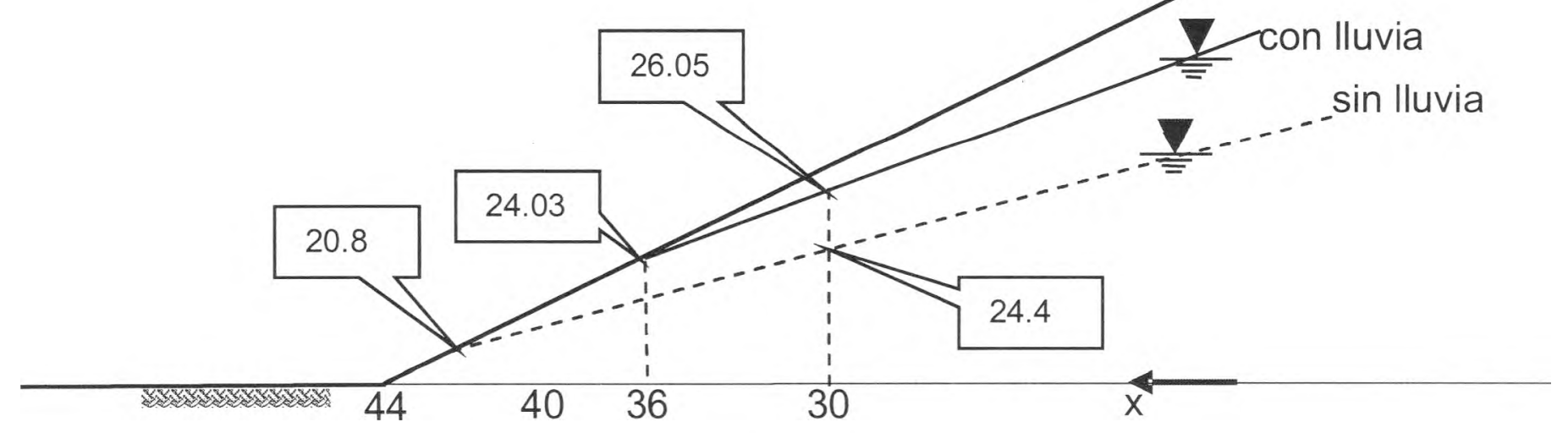

Figura 3 
Aplicando la expresión (8) se obtienen los valores numéricos de $h(x, t)$ que permiten construir la figura 3 . De acuerdo con la figura 3 las líneas de superficie de corriente, sin y con lluvia, cortan el talud de aguas abajo del bordo izquierdo respectivamente a $0.8 \mathrm{~m}$ y $4 \mathrm{~m}$ de altura por encima de su base.

El ascenso de la línea superior de corriente, por efecto de las lluvias, ocasiona una modificación de las fuerzas de filtración, así como de los empujes de Arquímedes en el cuerpo de terraplén. Correlativamente, se generan desplazamientos horizontales del bordo izquierdo al presentarse la estación pluviosa. La magnitud de los desplazamientos puede determinarse mediante el uso del elemento finito.

\section{Orden de magnitud de los desplazamientos inducidos por la lluvia}

A falta de datos precisos sobre el comportamiento mecánico del material constitutivo de los bordos, se trata únicamente de valorar el orden de magnitud de los desplazamientos inducidos por la lluvia. En consecuencia, se utilizará un método burdo de cuantificación.

El incremento de las fuerzas actuantes se debe:

a) al empuje de Arquímedes actuante sobre el volumen $\mathrm{AA}^{\prime} \mathrm{O}$ (Figura 4) limitado por las líneas superiores de corriente $\mathrm{AO}$ (sin lluvia) y $\mathrm{A}^{\prime} \mathrm{O}$ (con lluvia). Esta fuerza es vertical igual a 20 ton y su punto de aplicación es el centro de gravedad $\mathrm{G}$ del triangulo $\mathrm{AA}^{\prime} \mathrm{O}$. b) a las fuerzas de filtración actuantes en este mismo triangulo $\mathrm{AA}^{\prime} \mathrm{O}$ e iguales a 1.6 ton, en sentido vertical y hacia el interior del terraplén, y 5.3 ton en sentido horizontal y hacia aguas abajo. Su punto de aplicación es el centro de gravedad $\mathrm{G}$ del triangulo $\mathrm{AA}^{\prime} \mathrm{O}$ (Figura 4).

En estas condiciones, la fuerza total resultante es 19.1 ton; su punto de aplicación es cercano a la superficie del talud de aguas abajo y su dirección es cercana a la normal a este mismo talud.

Para valorar el orden de magnitud del desplazamiento inducido por esta fuerza resultante, se utiliza la solución elástica de Flamant (Love, 1944) suponiendo que esta fuerza está aplicada en la superficie de un medio semi-infinito y que se trata de un estado plano de deformaciones.

En la cercanía del punto de aplicación de la fuerza el desplazamiento normal a la cara del talud y hacia fuera esta dado por,

$$
V=\frac{2 F(1+v)}{\pi E}\left[\frac{1}{2}+(1-v) \ln (r)\right]
$$

y el desplazamiento tangente al talud y hacia el punto A es,

$$
U=\frac{F(1+v)}{2 E}(1-2 v)
$$

Con

$\mathrm{F}=19$ ton

$\mathrm{E}=500 \mathrm{ton} / \mathrm{m}^{2}$

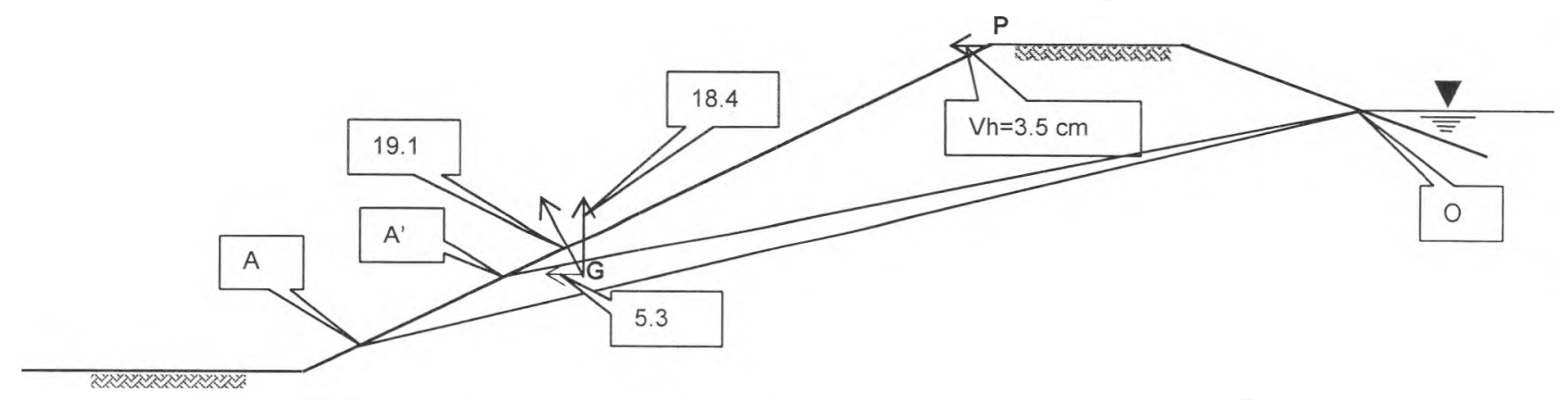

Figura 4 
$v=0.3$

$r=$ Distancia, en $\mathrm{m}$, entre el punto de aplicación

de la carga y el punto $P$, en que se desea conocer el desplazamiento. Se toma $\mathrm{r}=16 \mathrm{~m}$ que es la distancia $\overline{A^{\prime} P}$ (Figura 4).

Para el desplazamiento horizontal y hacia aguas abajo del punto $P$ :

$$
v_{h}=U \cos (\gamma)+V \operatorname{sen}(\gamma)=3.5 \mathrm{~cm}
$$

Como resultado, los desplazamientos horizontales máximos medidos en el bordo izquierdo del Canal de la Compañía son del mismo orden que los calculados con base en propiedades mecánicas supuestas pero razonables.

Convendrá obtener a futuro las propiedades mecánicas de los materiales constitutivos de los bordos mediante experimentos (pruebas de placa por ejemplo).

\section{Apéndice 1}

REM

REM

REM

DIM T $(300), E(300)$

CLS

$\mathrm{PI}=3.14159$

REM

REM

REM

DATA "CASO 3: LA COMPAÑIA"

DATA $31.7,20.0,33.0,26.6,21.8,6.0$

READ CASO\$

READ H1, H2, H3, GAMMA1, GAMMA2, LO

GAMMA $1=$ GAMMA $1 * \mathrm{PI} / 180$

GAMMA2 $=$ GAMMA $2 * \mathrm{PI} / 180$

REM

REM

REM

REM

REM

REM

REM

REM

REM

REM

REM

REM

REM
DATA 180,180

READ T0,T1

\section{LECTURA DE DATOS DE LLUVIA...}

ASIgnA A LA VARIABLE "DATLLUVIA\$", EL NOMBRE DEL ARCHiVo

DONDE SE ENCUENTRA GUARDADO, FISICAMENTE EN DISCO, EL REGISTRO TABULAR DE TIEMPOS-INTENSIDADES DE LLUVIA...

INPUT "NOMBRE DEL ARCHIVO TIEMPOS-INTENSIDADES DE LLUVIA"; DATLLUVIA\$

ABRE, EN DISCO, EL ARCHIVO ASIGNADO A LA VARIABLE "DATLLUVIA\$”...

OPEN "I", \#1, DATLLUVIA\$

LECTURA DEL REGISTRO DE TIEMPO-INTENSIDADES DE LLUVIA, 
DEL ARCHIVO ASIGNADO A LA VARIABLE "DATLLUVIA\$”"...

REM

FOR I $=1$ TO TO

INPUT \#1, T (I), E (I), BETA (I)

NEXT I

CLOSE \# 1

REM

LECTURA DE DATOS DEL SUELO...

REM

DATA $0.6,0.086$

READ N, K

OPEN "O", \#1, "CASO6. RES"

PRINT \#1, "<<"; CASO\$;" >>"

PRINT \#1, " "

PRINT\#1, " $* * * *$ DATOS GEOMETRICOS $* \star * * "$

PRINT \#1, " "

PRINT \#1, "H1, H2, H3, GAMMA1, GAMMA2, LO"

PRINT \#1, USING "\#\#\#\#.\#\#,"; H1,H2,H3, GAMMA1*180/PI, GAMMA2*180/PI, LO

PRINT \#1, " "

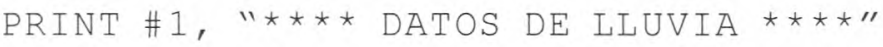

$\operatorname{PRINT} \# 1, " \mathrm{~T}(I), \mathrm{E}(I), \operatorname{BETA}(I) "$

FOR $I=1 \mathrm{TO} \mathrm{TO}$

PRINT \#1, T(I), E (I), BETA (I)

NEXT I

PRINT \#1, " "

PRINT \#1, "BETA, T0, T1"

PRINT \#1, USING “\#\#\#\#.\#\#\#\#,"; T0, T1

PRINT \#1,

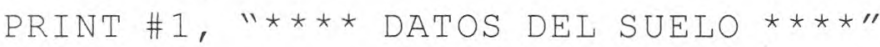

PRINT \# 1 , '

PRINT \#1, "N, K"

PRINT \#1, USING "\#\#\#\#.\#\#\#\#,"; N, K

$\mathrm{TGI}=1 / \mathrm{TAN}(\mathrm{GAMMA} 1)$

$\mathrm{TG} 2=1 / \mathrm{TAN}(\mathrm{GAMMA} 2)$

$\mathrm{M}=(\mathrm{H} 1-\mathrm{H} 2) * \mathrm{TG} 2$

$\mathrm{D}=(\mathrm{H} 3-\mathrm{H} 2) *(\mathrm{TG} 1+\mathrm{TG} 2)+\mathrm{L} 0-0.7 * \mathrm{M}$

$\mathrm{YO}=\mathrm{SQR}\left(\left(\mathrm{H} 1^{\wedge} 2-\mathrm{H} 2^{\wedge} 2\right)+\mathrm{D}^{\wedge} 2\right)-\mathrm{D}$

$\mathrm{L}=\mathrm{D}+\mathrm{YO} / 2$

$\mathrm{HM}=(\mathrm{H} 1+\mathrm{H} 2) / 2$

FOR $X=0$ TO D STEP D/10

$S 2=0$

FOR I $=0$ TO $(\mathrm{T} 1-1)$ STEP 1

$\operatorname{BETA}(I)=\operatorname{BETA}(I) * \mathrm{PI} / 180$

$S=0$

$\mathrm{E} 1=\mathrm{E}(\mathrm{T} 1-\mathrm{T}(\mathrm{I})) * \operatorname{COS}(\mathrm{BETA}(\mathrm{I})-\mathrm{GAMMA} 1) / \operatorname{COS}(\mathrm{GAMMA} 1)$

$I F I=0$ THEN GOTO 40 
$R=2 * S Q R(K * H M *(T(I)) / N)$

FOR NK $=0$ TO 10

$\mathrm{XI}=(\mathrm{X}+\mathrm{NK} \star \mathrm{L}) / \mathrm{R}$

IF XI $>=2.5$ THEN ERFCI $=0$

IF $X 1>=2.5$ THEN GOTO 20

$\mathrm{B}=\mathrm{X} 1$

GOSUB 10

$\mathrm{ERFC} 1=1-\mathrm{XI}$

$20 \quad \mathrm{X} 2=((\mathrm{NK}+1) \star \mathrm{L}-\mathrm{X}) / \mathrm{R}$

IF $X 2>=2.5$ THEN ERFC2 $=0$

IF $X 2>=2.5$ THEN GOTO 30

$\mathrm{B}=\mathrm{X} 2$

GOSUB 10

$\mathrm{ERFC2}=1-\mathrm{XI}$

$30 S=((-1) \wedge N K) *(E R F C 1+E R F C 2)+S$

NEXT NK

$40 \quad \mathrm{~S} 1=2 * \mathrm{E} 1 *(\mathrm{HM} / \mathrm{N}) *(1-\mathrm{S})$

$\mathrm{S} 2=\mathrm{S} 2+\mathrm{S} 1$

NEXT I

$\mathrm{H}=\mathrm{SQR}\left(\mathrm{S} 2+\mathrm{H} 1^{\wedge} 2-\left(\mathrm{H} 1^{\wedge} 2-\mathrm{H} 2^{\wedge} 2\right) * \mathrm{X} / \mathrm{L}\right)$

IF $X=0$ THEN PRINT \#1," "

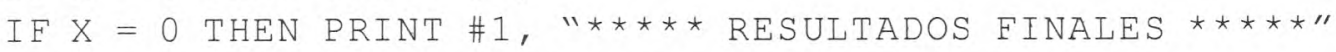

IE $X=0$ THEN PRINT \#1," "

IF $X=0$ THEN PRINT \# 1 , "TI, X, H"

PRINT \#1, USING “\#\#\#\#.\#\#\#\#,"; T1, X, H

NEXT X

$\operatorname{PRINT} \# 1$, " "

CLOSE \#1

END

1OREM

REM

REM CALCULO DE LA FUNCION ERROR...

REM

REM

$\operatorname{DIM} X A(20), W(20)$

$\operatorname{DEF} \operatorname{FNF}(X X)=(2 / S Q R(P I)) \star \operatorname{EXP}(-X X * X X)$

$\mathrm{NN}=10$

$\mathrm{XA}(6)=0.1488743389$

$\mathrm{XA}(7)=0.4333953941$

$\mathrm{XA}(8)=0.6794095682$

$X A(9)=0.8650633666$

$\mathrm{XA}(10)=0.9739065285$

$W(6)=0.2955242247$

$W(7)=0.2692667193$

$W(8)=0.2190863625$

$W(9)=0.1494513491$ 


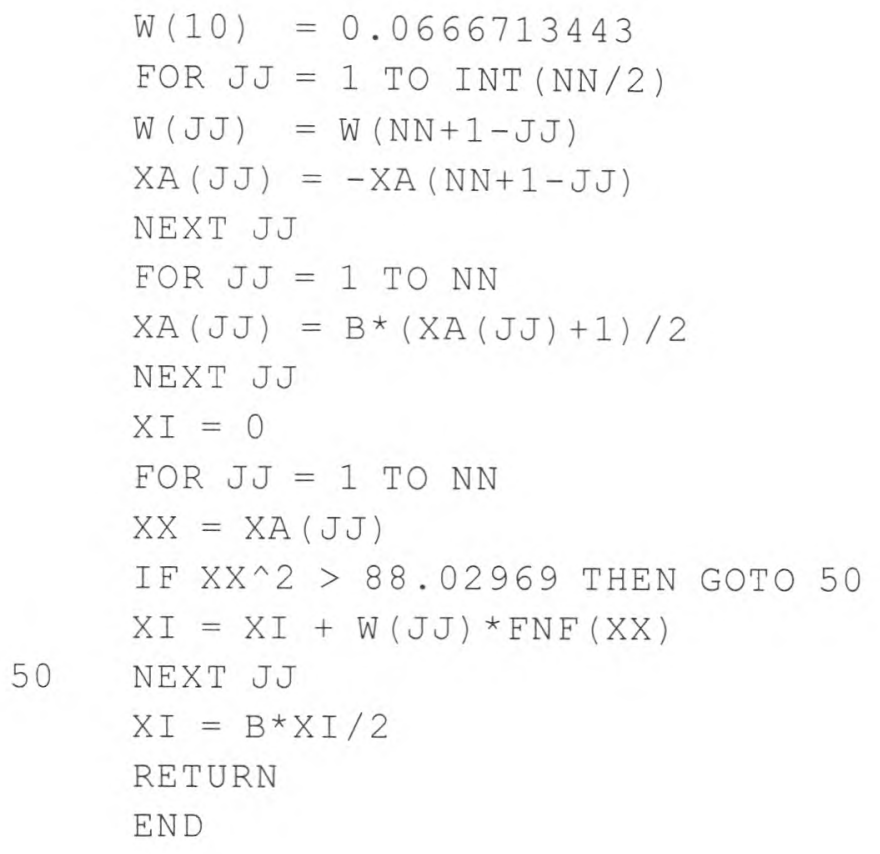

\section{Conclusiones}

La diferencia entre los desplazamientos horizontales medidos en los bordos izquierdo y derecho del Canal de la Compañía, puede deberse al efecto de las lluvias.

La inclinación de la lluvia respecto a la vertical ocasiona aportes variables de agua en los taludes externos de ambos bordos. Consecuentemente, las líneas superiores de corriente en el cuerpo de a mbos bordos resultan diferentes. El orden de magnitud de los desplazamientos calculados en el talud más expuesto a la lluvia da cuenta de los desplazamientos medidos.

\section{Referencias}

Carslaw H.S. y Jaeger J.C. (1959). Conduction of Heat in solids. Oxford, Clarendon Press, No. 11, p. 494.

CFE (2001). Instalación, equipamiento y medición de la instrumentación del canal Río de la Compañía. Informe 841-01-148/01.

Love A.E.H. (1944). A Treatise on the Mathematical Theory of Elasticity. Dover Publications, New York.

Polubarinova-Kochina P. (1962). Theory of Ground Water Movement. Princeton, Ed, Princeton N.J.

\section{Semblanza de los autores}

Jesús Alberro-Aramburu. Ingeniero civil egresado de la "Ecole Nationale de Ponts et Chaussées", de Paris, Francia en 1959. Estudió su maestría y doctorado en la División de Estudios de Posgrado de la Facultad de Ingeniería, UNAM de 1959 a 1963. Es Investigador titular del Instituto de Ingeniería desde 1964. Ha recibido diversas distinciones como el Premio Universidad Nacional Autónoma de México, Premio Javier Barros Sierra y Nabor Carrillo del Colegio de Ingenieros Civiles de México. Actualmente es investigador emérito del Sistema Nacional de Investigadores.

Rogelio R. Hernández-Hernández. Ingeniero civil egresado con mención honorífica de la Facultad de Ingeniería, UNAM. Realizó su maestría y doctorado en la División de Estudios de Posgrado de la Facultad de Ingeniería, UNAM. Es investigador del Instituto de Ingeniería y profesor en la Facultad de Ingeniería (Departamento de Matemáticas Aplicadas y Cálculo), así como de la División de Estudios de Posgrado de la Facultad de Ingeniería (Coordinación de Mecánica de Suelos). Actualmente es miembro del Sistema Nacional de Investigadores. 